\title{
FIPS: An R Package for Biomathematical Modelling of Human Fatigue Related Impairment
}

\author{
Micah Kate Wilson ${ }^{*}$, Luke Strickland ${ }^{1}$, and Timothy Ballard ${ }^{2}$ \\ ${ }^{1}$ Future of Work Institute, Curtin University \\ ${ }^{2}$ School of Psychology, University of Queensland
}

FIPS can accessed via the Github repository https://github.com/humanfactors/FIPS

\section{Summary}

In many workplace contexts, accurate predictions of a human's fatigue state can drastically improve system safety. Biomathematical models of fatigue (BMMs) are a family of dynamic phenomenological models that predict the neurobehavioural outcomes of fatigue (e.g., sleepiness, performance impairment) based on sleep/wake history (Dawson, Darwent, \& Roach, 2017). However, to-date there are no open source implementations of BMMs, and this presents a significant barrier to their broadscale a doption by researchers and industry practitioners.

FIPS is an open source $R$ package ( $R$ Core Team, 2020) to facilitate BMM research and simulation. FIPS has implementations of several published bio-mathematical models and includes functions for easily manipulating sleep history data into the required data structures. FIPS also includes default plot and summary methods to aid model interpretation. Model objects follow tidy data conventions (Wickham, 2014), enabling FIPS to be integrated into existing research workflows of $\mathrm{R}$ users.

\section{Background on Biomathematical Models}

Borbély's (1982) seminal two-process BMM specifies that subjective fatigue is modulated by the additive interaction of two biological processes: the homeostatic and the circadian. The homeostatic process, denoted by $S$, is responsible for the increase in fatigue during wake and the recovery from fatigue during sleep. Fluctuations in process $S$ are described by exponential functions with fixed lower and upper a symptotes. The endogenous circadian process, denoted

*Correspondence: Address correspondence to Michael David Wilson at michael.d.wilson@curtin.edu.au 
by $C$, reflects the effect of the body clock on sleep propensity. The dynamics of these processes are driven by a set of governing parameters (e.g., the phase of the circadian process). Figure 1 below shows the additive effects of varying governing parameters of $\mathrm{S}$ and $\mathrm{C}$. This model has formed the basis of many other models that predict neurobehavioural performance and fatigue based on sleep history (Åkerstedt, Ingre, Kecklund, Folkard, \& Axelsson, 2008; Hursh et al., 2004; Peng, Bouak, Wang, Chow, \& Vartanian, 2018; Ramakrishnan, Wesensten, Balkin, \& Reifman, 2016).

BMMs have a rich history of application in laboratory sleep deprivation studies where they are used to understand the latent factors underlying human fatigue. An important aim of these studies is to identify the governing parameter values which provide the best account for the data at hand (Reifman \& Gander, 2004). Further, propriety BMM implementations are frequently applied in safety-critical industries (e.g., aviation, military, mining operations) to support system safety as part of broader fatigue management strategies (e.g., aiding in rostering decisions; see Dawson et al., 2017; Dawson, Noy, Härmä, Åkerstedt, \& Belenky, 2011).

Unfortunately, the broader adoption of BMMs by the cognitive and behavioral sciences has been constrained by several factors. Firstly, BMM researchers have typically only provided the mathematical derivations of their models (i.e., formulae) and not their computational implementations. This is a barrier to reproducibility (Wilson, Boag, \& Strickland, 2019) because implementing BMMs and the required data structures from the ground up requires substantial expertise and time investment. Prior to FIPS, the only available BMM implementations were contained within closed-source commercial software (e.g., SAFTE-FAST; Hursh et al., 2004). Even for researchers and practitioners able to afford licenses, these tools prohibit users from inspecting, modifying, independently evaluating, or extending the code and contained models.

\section{Package Motivation and Features}

FIPS aims to make BMM approaches accessible to a wider community and assist researchers in conducting robust and reproducible biomathematical modelling of fatigue. The package includes:

- Functions to transform common sleep data formats into the longitudinal data structure required for conducting BMM simulation and estimation.

- Well documented implementations of three forms of BMM: The Unified Model (Ramakrishnan et al., 2016) and Two- and Three-Process Models (Åkerstedt et al., 2008; Borbely, 1982; Reifman \& Gander, 2004).

- A function for plotting BMM outputs, including with observed data points. The visualisations are publication-ready, but flexibly adjusted via the ggplot2 package.

The package also contains two vignettes: a walk-through of a sleep simulation scenario which 


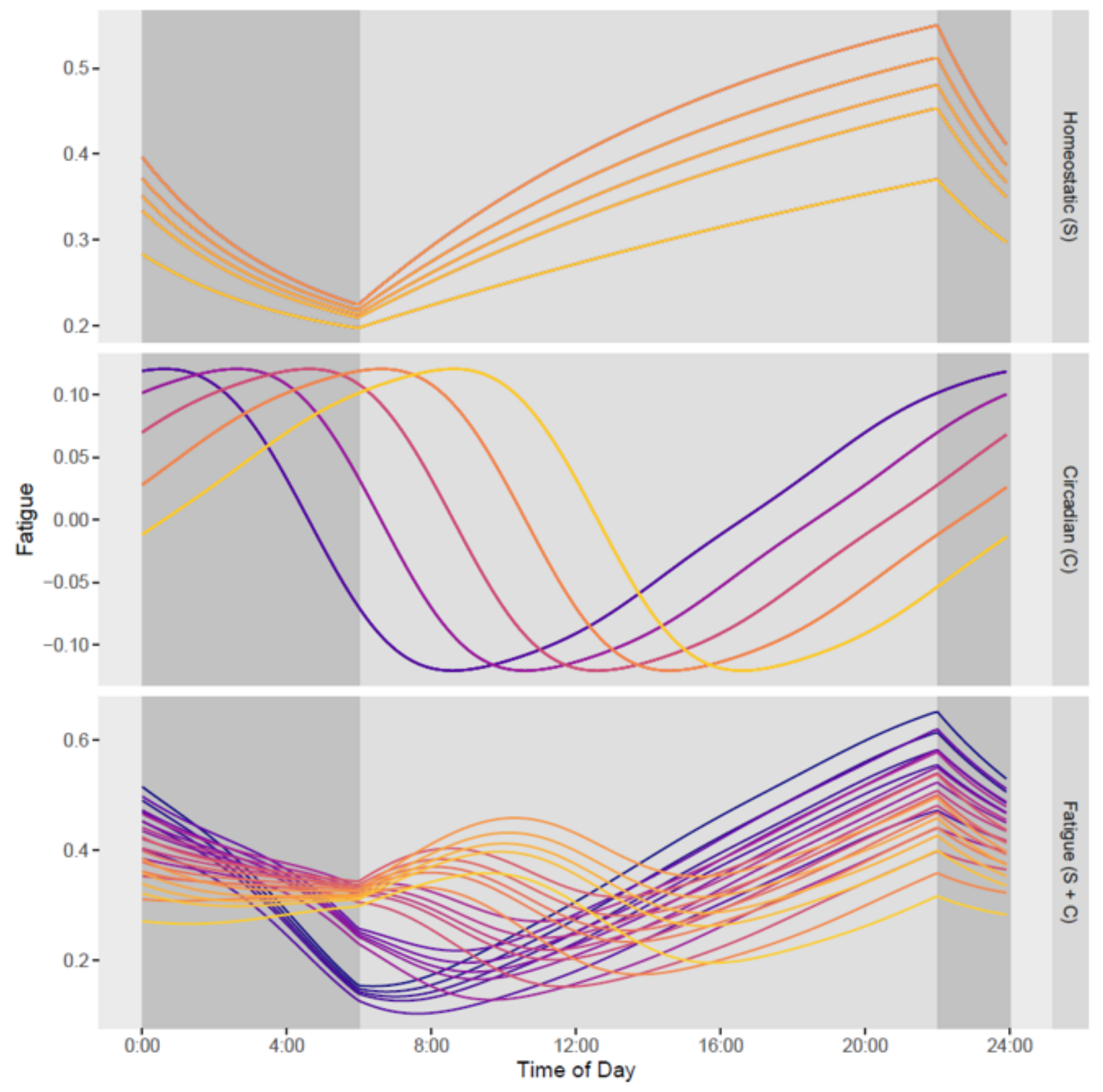

Figure 1: A parameter sensitivity plot of the Three Process Model. The $x$ axis represents a 24 hour day, with the dark gray plot regions indcating sleep and the light gray indicating wake. The top panel shows the homeostatic process with five variations of the $\tau_{d}$ parameter and the centre panel shows the circadian process with five variations of the $\varphi_{\text {phase }}$ parameter. The bottom panel shows the multiplicative combinations of all unique $\mathrm{S}$ and $\mathrm{C}$ processes from the previous panels. The plot was produced with functionality in FIPS. 
includes generating, transforming and analyzing the data; and a detailed tutorial in plotting model outputs.

\section{FIPS Interface and Data Structures}

Conducting a BMM simulation in FIPS requires users to generate a FIPS_df, a tidy data frame containing a time series (based on sleep history) of all variables required to conduct BMM research. FIPS supports two sleep data formats, each format associated with a corresponding function that automatically performs all required transformations to the FIPS_df format:

- The parse_sleeptimes function transforms a data frame containing three vectors: sleep onset times, sleep end times (i.e., awakening), and the sleep episode sequence identifier. This format is human readable and well suited for individuals who are manually entering sleep history data (e.g., from a paper sleep diary). ${ }^{1}$

- The parse_sleepwake_sequence function transforms a bit vector representing sleep (0) and wake (1) statuses, with each bit representing an equal epoch (e.g., 1 minute). While not very human readable, this data format is commonly output by actigraphy devices and their corresponding sleep/wake status algorithms (e.g., Cole-Kripke). Consequently, this format is often supported by other proprietary BMM software.

The resulting FIPS_df dataframe can then be sent to the simulation dispatch function FIPS_simulate to execute a specific model. This function requires: a FIPS_df, a model string (e.g., "unified"), and an associated parameter vector for the selected model (pvec). Documentation is provided for customizing each parameter in the pvec, with citations for the default values. The returned FIPS_simulation data frame has added columns for each time-step of the series, including model predictions (e.g., alertness and sleepiness in the case of the three process model), as well as time-varying model processes (e.g., the circadian process, $c$, and homeostatic process, s). FIPS_simulation predictions can be plotted by calling plot or FIPS_plot on the object, and simulation and model configurations can be reviewed via summary or print.

\section{Research \& Future Development}

FIPS is being actively developed as part of a broader research project examining cognitive fatigue prediction in safety-critical workplace environments. Given the extensibility of FIPS's implementation, we hope other BMM researchers may consider collaborating to implement other BMMs in the framework.

\footnotetext{
${ }^{1}$ It should be noted that the parse_sleeptimes function may also be useful to users wishing to convert a set of human-readable sleep times (e.g., manually entered from a sleep diary) to a bit vector (i.e., a list of 1 or 0 values). This is because the FIPS_df contains a column representing the series as a bit vector (see FIPS_df $\$$ wake_status_int).
} 


\section{Acknowledgments}

The Future of Work Institute at Curtin University supported the development of this package.

\section{License}

This project is licensed under the "GNU Affero General Public License" version 3 - see the LICENSE file for details

\section{References}

Åkerstedt, T., Ingre, M., Kecklund, G., Folkard, S., \& Axelsson, J. (2008). Accounting for partial sleep deprivation and cumulative sleepiness in the three-process model of alertness regulation. Chronobiology International, 25(2-3), 309-319. doi:10.1080/07420520802110613

Borbely, A. A. (1982). A two process model of sleep regulation. Human Neurobiology, 1, 195-204.

Dawson, D., Darwent, D., \& Roach, G. D. (2017). How should a bio-mathematical model be used within a fatigue risk management system to determine whether or not a working time arrangement is safe? Accident Analysis and Prevention, 99, 469-473. doi:10.1016/j.aap.2015.11.032

Dawson, D., Noy, Y. I., Härmä, M., Åkerstedt, T., \& Belenky, G. (2011). Modelling fatigue and the use of fatigue models in work settings. Accident Analysis 8 Prevention, 43(2), 549-564. doi:10.1016/j.aap.2009.12.030

Hursh, S. R., Redmond, D. P., Johnson, M. L., Thorne, D. R., Belenky, G., Balkin, T. J., Storm, W. F., et al. (2004). Fatigue models for applied research in warfighting. Aviation, space, and environmental medicine, 75(3), A44-A53.

Peng, H. T., Bouak, F., Wang, W., Chow, R., \& Vartanian, O. (2018). An improved model to predict performance under mental fatigue. Ergonomics, 61(7), 988-1003. doi:10.1080/00140139.2017.1417641

Ramakrishnan, S., Wesensten, N. J., Balkin, T. J., \& Reifman, J. (2016). A unified model of performance: Validation of its predictions across different sleep/wake schedules. Sleep, 39(1), 249-262. doi:10.5665/sleep.5358

R Core Team. (2020). R: A language and environment for statistical computing. Vienna, Austria: R Foundation for Statistical Computing. Retrieved from https://www.R-project.org/

Reifman, J., \& Gander, P. (2004). Commentary on the three-process model of alertness and broader modeling issues. Aviation, space, and environmental medicine, 75(SUPPL.1), A84-8; discussion A89. 
Wickham, H. (2014). Tidy data. Journal of Statistical Software, 59(10). doi:10.18637/ jss.v059.i10

Wilson, M. K., Boag, R. J., \& Strickland, L. (2019). All models are wrong, some are useful, but are they reproducible? Commentary on lee et al. (2019). Computational Brain 83 Behavior, 2(3-4), 239-241. doi:10.1007/s42113-019-00054-x

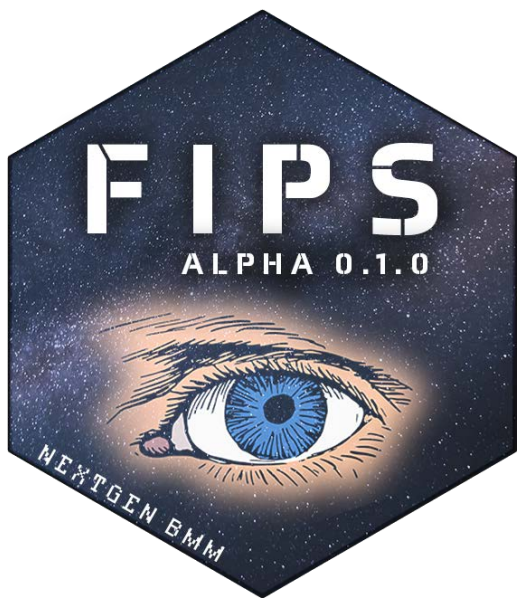

